\title{
The Dark Side of EDX Tomography: Modeling Detector Shadowing to Aid 3D Elemental Signal Analysis
}

\author{
Catriona S.M. Yeoh, ${ }^{\star}$ David Rossouw, Zineb Saghi, Pierre Burdet, Rowan K. Leary, and \\ Paul A. Midgley
}

Department of Materials Science and Metallurgy, University of Cambridge, 27 Charles Babbage Road, Cambridge, CB3 OFS, UK

\begin{abstract}
A simple model is proposed to account for the loss of collected X-ray signal by the shadowing of X-ray detectors in the scanning transmission electron microscope. The model is intended to aid the analysis of threedimensional elemental data sets acquired using energy-dispersive X-ray tomography methods where shadow-free specimen holders are unsuitable or unavailable. The model also provides a useful measure of the detection system geometry.
\end{abstract}

Key words: EDX tomography, detector shadowing, elemental analysis

\section{INTRODUCTION}

Energy-dispersive X-ray (EDX) microanalysis is a valuable materials characterization technique used in a broad range of industrial and academic fields (Reed, 1975; Goldstein, 1979; Williams \& Carter, 2009; Watanabe, 2011). Until recently, a major limitation of EDX microanalysis in the scanning transmission electron microscope (STEM) has been the low signal collection efficiency, with typically $1 \%$ or less of all X-rays generated by the electron beam reaching the detector (Schlossmacher, 2010; Watanabe, 2011). Such low collection efficiencies have made elemental mapping a very slow process in the STEM. Nowadays, however, the latest generation of STEMs are equipped with EDX systems with much improved signal collection efficiencies, in large part owing to the introduction of silicon drift detectors (SDDs), increased detector areas (Newbury, 2005), and the use of multiple detectors (von Harrach et al., 2010). The Super-X system used in this study, for example, has four SDDs placed symmetrically around the optic axis, with a total solid angle of $0.9 \mathrm{sr}$, resulting in approximately one order of magnitude improvement in collection efficiency compared with traditional systems. These modern systems have reduced the acquisition time and electron dose required to acquire high spatial resolution two-dimensional (2D) elemental maps with good signal-to-noise ratios.

Electron tomography is now well established in the physical sciences for the 3D structural characterization of materials (Midgley \& Weyland, 2003; Midgley \& DuninBorkowski, 2009; Leary et al., 2012), and the extension to 3D elemental mapping via EDX tilt series is an important goal. Early attempts (Möbus et al., 2003; Saghi et al., 2007) suffered from low signal collection efficiencies owing to the use of a single detector located on one side of the sample. However, the introduction of improved EDX instrumentation has inspired a renaissance in EDX tomography,

Received September 1, 2014; accepted February 3, 2015

${ }^{*}$ Corresponding author. csmy2@cam.ac.uk evidenced by a growing number of studies appearing in the literature, including the 3D elemental mapping of a $28 \mathrm{~nm}$ metal gate transistor (Lepinay et al., 2013), a lithium ion battery cathode (Genc et al., 2013), and hollow metal nanoparticles (Goris et al., 2014; Slater et al., 2014b).

Inevitably, there will be a range of tilt angles over which $\mathrm{X}$-rays emitted from a region of interest will be partially or completely obstructed by objects surrounding the region. This is especially so when needle specimens or dedicated low-profile EDX holders are not available, as there may be substantial shadowing by the specimen holder and/or the supporting grid. Although the Super-X system (von Harrach et al., 2010) employs multiple large SDD detectors, these still experience partial or complete shadowing over an appreciable angular range. The shadow cast onto a detector results in a loss of collected X-ray signals, which should be accounted for when interpreting tomographic data sets. Indeed, nominal specimen holder tilts to minimize shadowing have been discussed (Zaluzec, 2014) and the issue of detector shadowing has been described as likely to compromise the reconstruction of quantitative tomographic X-ray spectroscopy data (Slater et al., 2014a).

To be suitable for tomographic reconstruction, the EDX intensity must vary at least monotonically with some physical property (Hawkes, 2006) and ideally would vary linearly with thickness and composition as in the thin film limit (Tixier \& Philibert, 1969). However, in an EDX tilt series data set, signal intensity variations owing to detector shadowing or changes in specimen thickness may be indistinguishable. Here, we present a simple model to predict and correct for EDX detector shadowing in an effort toward recovering elemental signal intensities suitable for quantitative $3 \mathrm{D}$ tomographic reconstruction.

\section{Model Description}

To accurately account for EDX detector shadowing, knowledge of the sample geometry, transmission electron microscope 


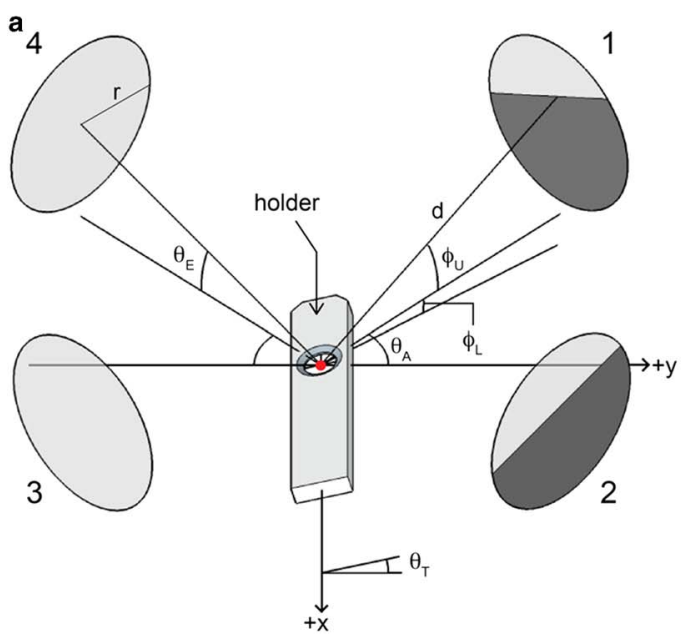

b

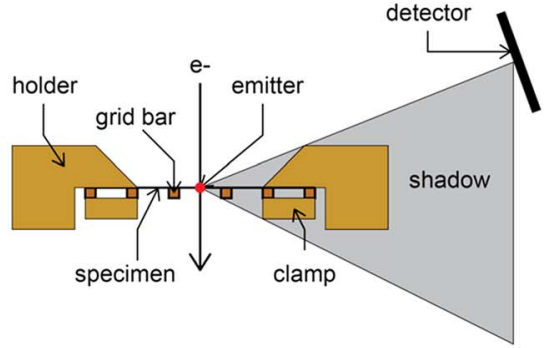

Figure 1. a: Three-dimensional representation of the partial shadowing of energy-dispersive X-ray (EDX) detectors 1 and 2 by a holder tilted to $\theta_{\mathrm{T}}=15^{\circ}$. Each EDX detector, of radius $r$, is located a distance $d$ from the X-ray source, at an elevation of $\theta_{\mathrm{E}}$ from the $x y$ plane perpendicular to the incident electron beam, and at an azimuthal angle of $\theta_{\mathrm{A}}$ from the $y$-axis, which is perpendicular to the tilt axis $x$. The upper and lower shadow angles $\phi_{\mathrm{U}}$ and $\phi_{\mathrm{L}}$ define the limits of height of the shadow cast on the detector $h$. b: Shadow angles $\phi_{\mathrm{U}}$ and $\phi_{\mathrm{L}}$ may be caused by a grid bar, clamp, or the holder itself.

(TEM) holder, supporting grid, and the location and geometry of the X-ray detector(s) is required. Precise information can be difficult to obtain and may vary from the manufacturer's specifications; although notably, it has been shown that the detector take-off angle may be determined from experimental EDX spectra containing coherent bremsstrahlung oscillations (Pantel, 2011). Arguably, a practical method to account for detector shadowing is to use a model with relatively few inputs to fit to an experimental data set. The model described here uses just two shadow angle inputs, an upper and lower shadow angle, to define the limits of the shadow. These two parameters, combined with the detector geometry, are used to characterize the shadowing of an EDX detector over a full sample tilt range (Fig. 1). It is important to note that the detector is assumed to be circular in this model. A detailed description of different detector geometries can be found elsewhere (Zaluzec, 2014).

An individual detector is described by the detector radius, $r$, the distance from the emitter to the detector, $d$, the elevation of the detector, $\theta_{\mathrm{E}}$, the angle to the bottom of the detector, $\theta_{\mathrm{D}}$, the azimuthal angle, $\theta_{\mathrm{A}}$, and the angle between the detector and the optic axis, $\delta$ (see Figs. 1,2). These are related according to the following equation. The true value of $\delta$, shown in Figure 2, is unknown and here we treat it as a free parameter to be fitted to the data sets:

$$
\begin{aligned}
& |\mathrm{CD}|=r \\
& |\mathrm{OC}|=d \\
& \frac{r}{\sin \left(\theta_{\mathrm{E}}-\theta_{\mathrm{D}}\right)}=\frac{d}{\sin \left(90+\theta_{\mathrm{D}}-\delta\right)} .
\end{aligned}
$$

The shadowing of individual circular detectors is based on calculation of the area of a partially filled circle. Foreshortening of this area due to the tilt is not expected to be significant as the detector is inclined toward the sample to maximize the solid angle. The shadow cast on the detector fills a varying

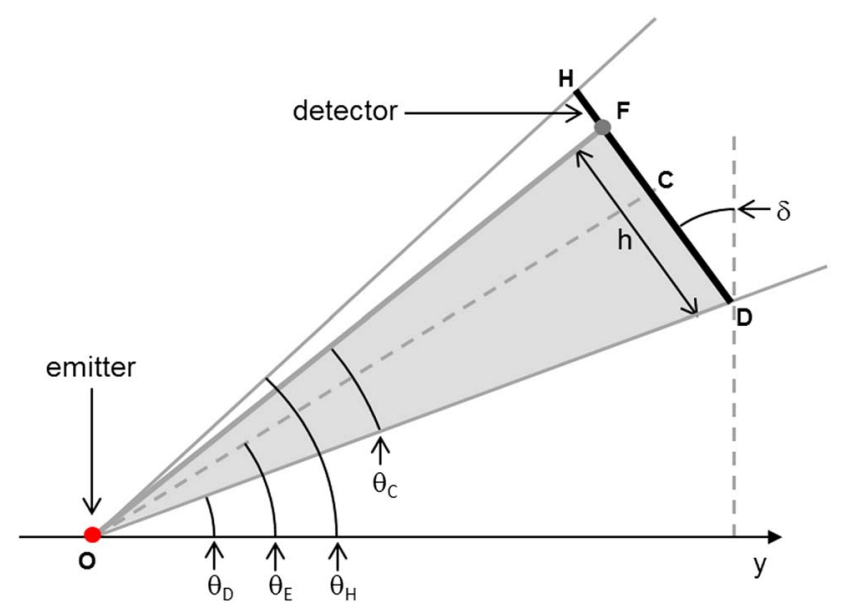

Figure 2. Diagram showing the plane containing the optic axis, the emitter (red circle), and the center of the detector; the shadowed detector is at an elevation of $\theta_{\mathrm{E}}$ (to the center of the detector) and at an angle $\delta$ to the vertical. The combined angle $\theta_{\mathrm{C}}$ describes the angular range of the shadow with height, $h$, on the detector, where $\mathrm{F}$ is the point where the shadow intersects the detector.

fraction of the circular detector area as a function of tilt. The angular range of the shadow is determined by the upper and lower shadowing angles, $\theta_{\mathrm{U}}$ and $\theta_{\mathrm{L}}$, and the holder tilt angle, $\theta_{\mathrm{T}}$.

The tilt angle $\theta_{\mathrm{T}}$ and the shadowing angles, which rotate about the tilt axis (parallel to the $x$-axis), must be combined and projected into the plane containing the detector, which is at an angle $\theta_{\mathrm{A}}$ to the $y$-axis (see Fig. 1), giving $\theta_{\mathrm{C}}$ [equation (2) and Fig. 2]:

$$
\theta_{\mathrm{C}}=\tan ^{-1}\left(\tan \left(\theta_{\mathrm{T}}+\phi_{\mathrm{U}, \mathrm{L}}\right) \cos \theta_{\mathrm{A}}\right)-\theta_{\mathrm{D}},
$$

where the angular origin has been changed such that detector shadowing begins at $\theta_{\mathrm{C}}=0$. The height, $h$, of the shadow's 


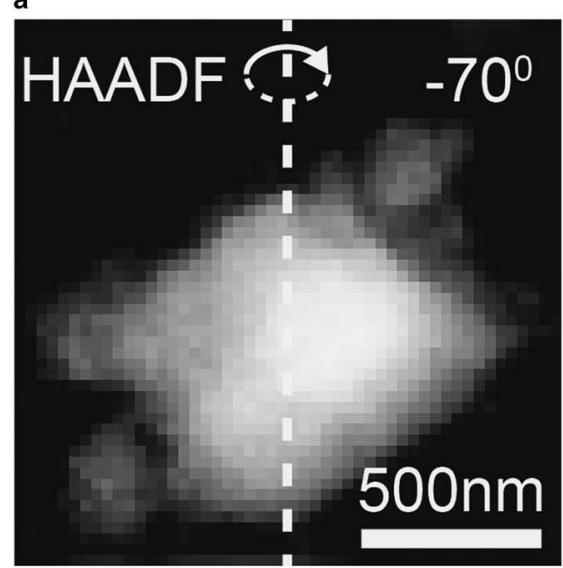

d

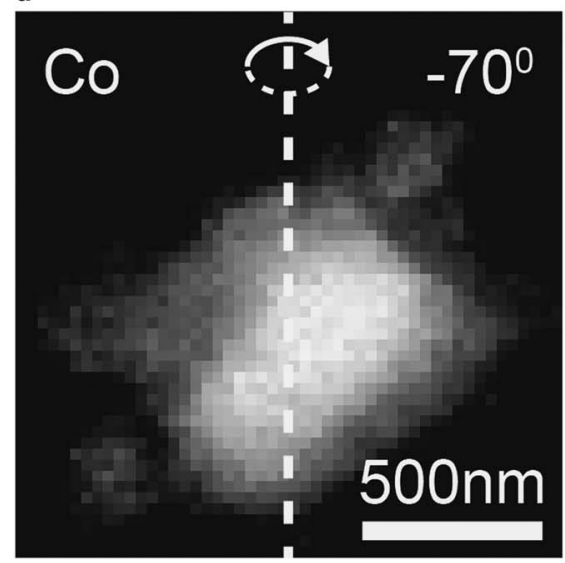

b

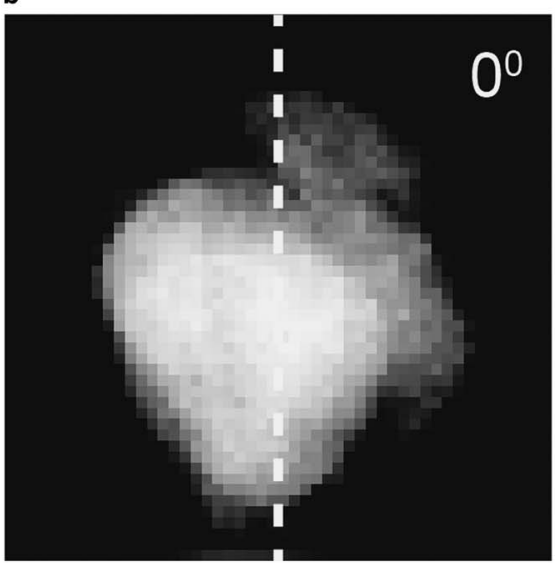

e

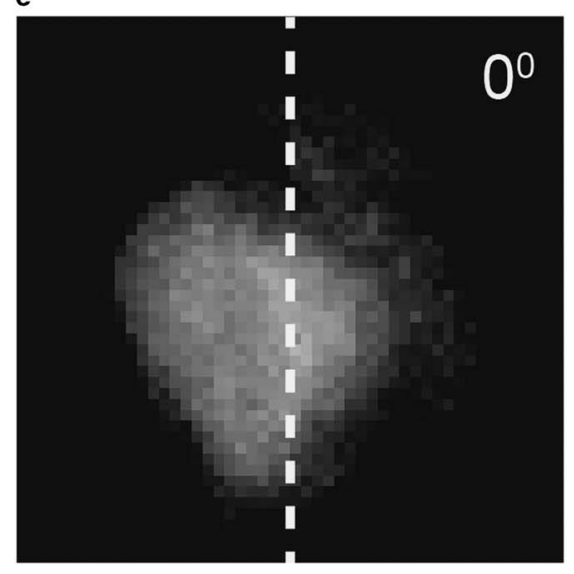

c

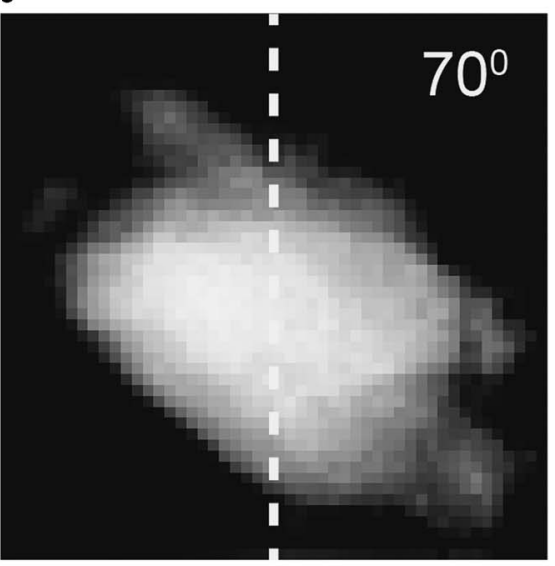

f

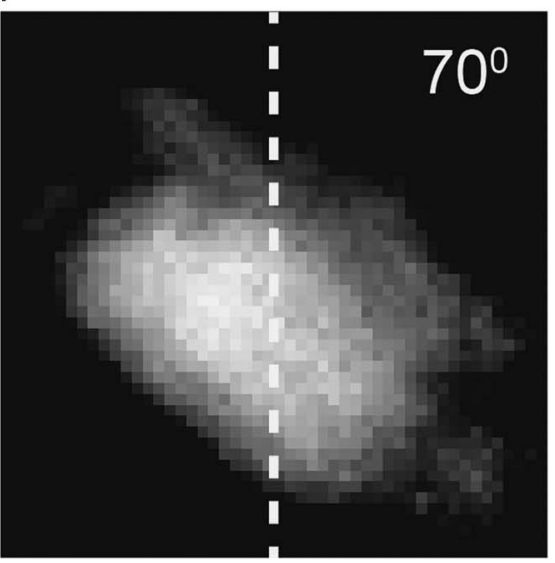

Figure 3. Selected high-angle annular dark-field (HAADF) scanning transmission electron microscopic images (a-c) and corresponding Co-K $\alpha$ X-ray intensity maps (d-f) from an acquired tilt series of an isolated $\gamma$ alumina powder containing cobalt oxide nanoparticles.

edge cast on a circular detector (see Fig. 2) may then be calculated according to the following equation:

$$
\begin{aligned}
|\mathrm{OD}| & =\frac{d}{\cos \left(\theta_{\mathrm{E}}-\theta_{\mathrm{D}}\right)} \\
\widehat{\mathrm{ODC}} & =180-\left(90-\theta_{\mathrm{D}}\right)-\delta \\
& =90+\theta_{\mathrm{D}}-\delta \\
\widehat{\mathrm{OFD}} & =180-\widehat{\mathrm{OBC}}-\theta_{\mathrm{C}} \\
& =90-\theta_{\mathrm{D}}+\delta-\theta_{\mathrm{C}} \\
h & =d \sec \left(\theta_{\mathrm{E}}-\theta_{\mathrm{D}}\right) \sin \theta_{\mathrm{C}} \csc \left(90-\theta_{\mathrm{D}}+\delta-\theta_{\mathrm{C}}\right) .
\end{aligned}
$$

When the shadow height on the detector is known, the shadowed area segment, $A$, of the detector is given by the following equation:

$$
A=r^{2} \cos ^{-1}\left(\frac{r-h}{r}\right)-(r-h) \sqrt{2 r h-h^{2}} .
$$

These equations can be readily fit to experimental data.

\section{Methods}

Experimental X-ray signals were acquired from a sample of porous $\gamma$ alumina-supported cobalt oxide nanoparticles, which was provided by Sasol (Sasol Ltd., Johannesburg, South Africa). ${ }^{a}$ The powdered sample was deposited on a conventional holey carbon 200-mesh copper grid, which was placed on a Fischione 2020 high tilt tomography holder (E.A. Fischione Instruments, Inc., Export, Pennsylvania, USA). EDX data were acquired with an FEI Osiris TEM (FEI Company, Hillsboro, Oregon, USA) equipped with a high brightness X-FEG gun (FEI Company) and a Super-X EDX system (FEI Company) comprising four detectors, each $30 \mathrm{~mm}^{2}$ in area and arranged symmetrically around an optic axis according to Figure 1. EDX maps were obtained from a region enclosing a powder fragment over a tilt range of -70 to $+70^{\circ}$ at $5^{\circ}$ increments, with the sample carefully positioned at eucentric height. As signals from individual detectors could not be separated postacquisition, at each tilt, X-ray data were acquired from detectors $1+2,3+4$, and all four detectors, resulting in the acquisition of $(3 \times 15=45)$ EDX maps over a 75 -min period.

The cobalt (Co) component of the sample emits highenergy X-rays at $6.9 \mathrm{keV}$. This $\mathrm{Co}-\mathrm{K} \alpha$ signal was obtained as an integrated peak area, which was background subtracted and normalized to the largest peak area in the tilt series. The highenergy Co-K $\alpha$ X-rays will suffer minimal absorption in the

\footnotetext{
${ }^{\mathrm{a}}$ www.sasol.com
} 
powdered sample. Possible Co-K $\alpha$ peaks from the pole pieces of the microscope were not observed and therefore any decrease in collected Co signal as a function of specimen tilt is assumed to be owing to detector shadowing only. Figure 3 displays selected high-angle annular dark-field STEM images and corresponding Co maps from the tilt series.

\section{Results}

Figure 4 displays the normalized intensity of the Co-K $\alpha$ X-ray peak extracted from each EDX map and plotted against the active detector fraction, defined as the detector fraction outside of the shadow predicted by the model. Note that the Co intensity at each tilt is summed over the whole sample shown in Figures $3 \mathrm{~d}$ to $3 \mathrm{f}$, as by summing the signal, the total volume generating the Co X-rays remains constant across the tilt series. The modeled detector geometry was defined by four circular detectors arranged symmetrically around an optic axis at azimuthal angles of $45,135,225$, and $315^{\circ}$, with each detector defined by a radius, distance, and elevation of $2.9,10.5 \mathrm{~mm}$, and $22^{\circ}$, respectively. The upper and lower shadow angle and the angle of the detector from the vertical, $\delta$, were subsequently refined to obtain a best fit to the experimental data using a least squares fitting. The data range used for fitting was limited to $\pm 50^{\circ}$, and an excellent fit was obtained using an upper shadowing angle of $22^{\circ}$ and a lower angle of $17^{\circ}$ in the emitterdetector plane. We find the model is reasonably insensitive to the value of $\delta$, but here it determines an optimum fit of $\delta=15^{\circ}$. As mentioned earlier, $\delta$ is expected to maximize the solid angle and so is not likely to deviate by more than $\sim 10^{\circ}$ from the value of $\theta_{\mathrm{E}}$ (where the detector is perpendicular to the elevation). The upper and lower shadowing determined by the model may be caused by either the sample holder or the grid bars in the supporting 200-mesh grid.

\section{Discussion}

As seen in Figure 4, close to half of the total detector area is in shadow at zero specimen tilt. Typically, to avoid detector shadowing, the user tilts the sample holder toward the EDX detector. However, in the multiple detector configuration used here, tilting toward a pair of detectors on one side of the tilt axis will inevitably cause the detectors on the opposite side of the tilt axis to enter into shadow. In fact, at approximately $\pm 25^{\circ}$ tilt, the difference in active detector area on either side of the tilt axis is equivalent to night and day. These observations confirm clearly that the variation in active detector area with tilt is substantial and that it is therefore important to consider the variation in detector shadowing across an EDX tilt series.

In addition to accounting for lost signal in a data set, the EDX shadowing model can also be used to identify optimum conditions for EDX tomography experiments. Figure 5 displays the effect of changing the upper and lower shadowing angles, the detector elevation, and the detector tilt on the active detective area across a full tilt range. Except where specified, a detector radius, distance, elevation, tilt, and

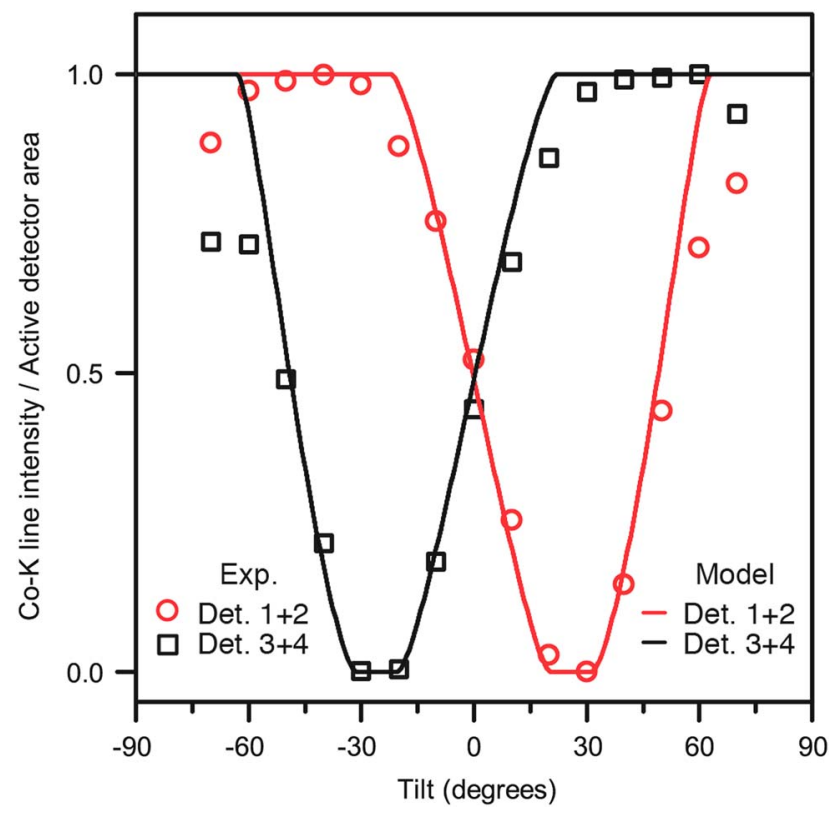

Figure 4. Experimental data of the integrated Co-K $\alpha$ intensity fitted with the shadowing model using upper and lower shadowing angles of $\phi_{\mathrm{U}}=22^{\circ}$ and $\phi_{\mathrm{L}}=17^{\circ}$, respectively, and a $\delta$ angle of $15^{\circ}$.

azimuth of $2.9,10.5 \mathrm{~mm}, 22,15$, and $45^{\circ}$ are used in the model, as used in the fitting above. In terms of shadowing angles, Figures $5 \mathrm{a}$ and $5 \mathrm{~b}$ show that as shadowing angles increase, the total active detector fraction across all tilts is reduced as expected. For example, the active detector fraction at $0^{\circ}$ is reduced from $100 \%$ with a $\pm 5^{\circ}$ shadow to $50 \%$ with a $\pm 20^{\circ}$ shadow. With regards to the detector elevation angle, $\theta_{\mathrm{E}}$, Figures $5 \mathrm{c}$ and $5 \mathrm{~d}$ show that while the total shadowing across all tilts is unchanged, shadowing at $0^{\circ}$ is significantly reduced when using higher elevations, and there is no shadowing at $0^{\circ}$ tilt for $45^{\circ}$ elevation. Figures $5 \mathrm{e}$ and $5 \mathrm{f}$ show that changing the angle of the detector, $\delta$, does not make a significant difference for this model, with the least shadowing occurring around a tilt of $10^{\circ}$.

It is clearly advantageous to use EDX systems with large detector elevation angles and specimen holders with low profiles in the direction of the detector(s). In practice, the maximum detector elevation angle is likely limited by the geometry of the upper objective pole piece. A further important consideration highlighted by the model, but depending more on the particular nature of the specimen, is the optimum strategy for combining the signals from the multiple detectors. This will be discussed in a future publication.

Care must be taken when using the model to find the upper and lower shadowing angles in a given data set. It is important to use high-energy X-ray peaks, which suffer the least absorption in the sample and the emitting volume must remain constant over the tilt range. Thin films, for example, do not meet the constant volume criterion. The shadowing angles in a data set are also sensitive to the position of the emitting volume. A region of interest positioned near the center of the sample holder and the grid bars (if a supporting mesh is used) 

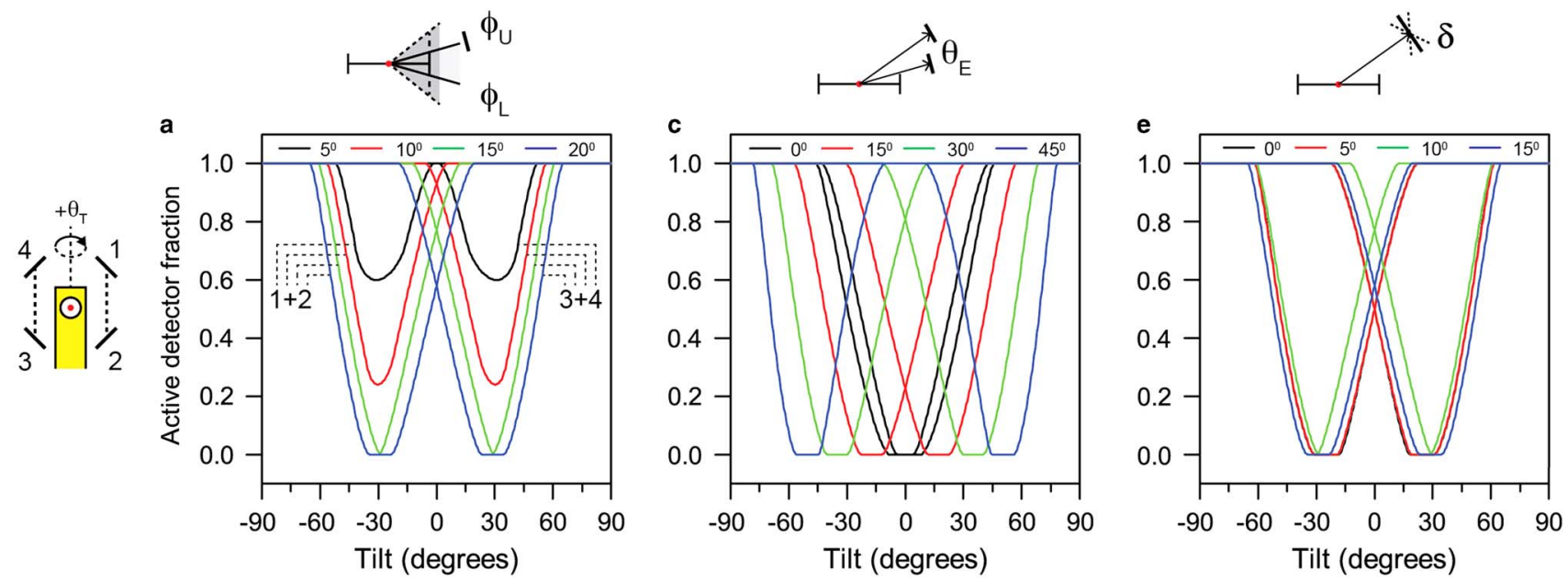

b

d

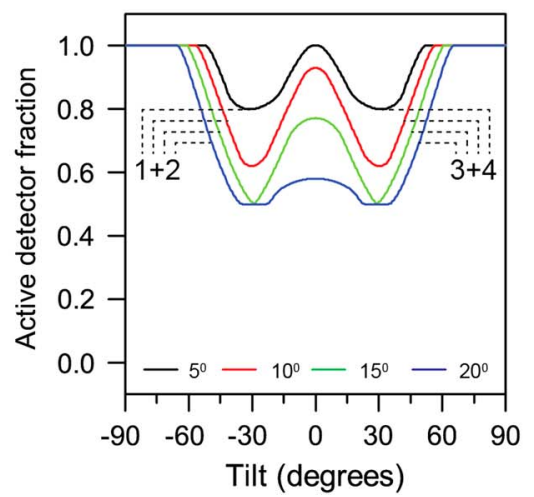

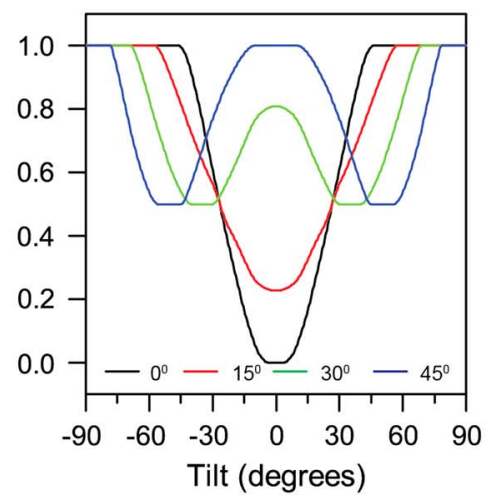

f

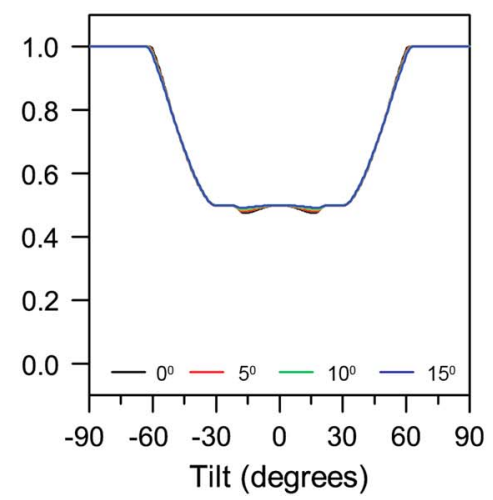

Figure 5. The effect of changing: (a, b) the upper and lower shadowing angles, (c, d) the detector elevation, and (e, f) the detector angle on the active detective area across a full tilt range. a,c,e: The individual active areas from detector pairs $1+2$ and $3+4$. b,d,f: The summed active areas from all four detectors.

should be used when applying the model; a significantly offset region of interest may result in asymmetrical shadowing requiring a different set of shadowing angles for each detector. The model can be fit to signals summed from all four detectors; however, for more accurate fitting, separating signals on either side of the tilt axis is recommended.

\section{CONCLUSIONS}

The EDX detector shadowing model described here provides a relatively quick and simple way to determine the loss of signal owing to blocked X-rays in the acquisition of an EDX tilt series for a powder sample dispersed on a grid with C-film, applicable to situations where shadowing is unavoidable. To decrease detector shadowing, significant benefit could be gained from redesigned tomographic holders with reduced thickness in the direction of the EDX detector(s). When a supporting mesh grid is used, the grid bars themselves will also cast a shadow, and in some cases their shadowing angles may be greater than those cast by the holder. To reduce the grid bar shadowing angles, a region close to the center of the grid should be chosen for analysis.

\section{ACKNOWLEDGMENTS}

The research leading to these results was funded by the European Research Council under the European Union's Seventh Framework Program (FP7/2007-2013)/ERC grant agreement 291522-3DIMAGE. In addition, D.R. acknowledges support from the Royal Society's Newton International Fellowship scheme. C.S.M.Y. acknowledges support from David Brown (Sasol St. Andrews and Sasol UK Ltd.) and R.K.L. acknowledges a Junior Research Fellowship at Clare College.

\section{REFERENCES}

Genc, A., Kovarik, L., Gu, M., Cheng, H., Plachinda, P., Pullan, L., FREITAG, B. \& WANG, C. (2013). XEDS STEM tomography for 3D chemical characterization of nanoscale particles. Ultramicroscopy 131, 24-32.

Goldstein, J.I. (1979). Principles of thin film X-ray microanalysis. In Introduction to Analytical Electron Microscopy, Hren J.J., Goldstein, J.I. \& Joy D.C. (Eds.), pp. 83-120. New York, NY and London: Springer.

Goris, B., Polavarapu, L., Bals, S., Van Tendeloo, G. \& Liz-MarZÁn, L.M. (2014). Monitoring galvanic replacement 
through three-dimensional morphological and chemical mapping. Nano Lett 14, 3220-3226.

HAWKes, P.W. (2006). The electron microscope as a structure projector. In Electron Tomography SE - 4, Frank, J. (Ed.), pp. 83-111. New York, NY: Springer.

Leary, R., Midgley, P.A. \& Thomas, J.M. (2012). Recent advances in the application of electron tomography to materials chemistry. Acc Chem Res 45, 1782-1791.

Lepinay, K., Lorut, F., Pantel, R. \& Epicier, T. (2013). Chemical 3D tomography of $28 \mathrm{~nm}$ high $\mathrm{K}$ metal gate transistor: STEM XEDS experimental method and results. Micron 47, 43-49.

Midgley, P.A. \& Dunin-Borkowski, R.E. (2009). Electron tomography and holography in materials science. Nat Mater 8, 271-280.

Midgley, P.A. \& Weyland, M. (2003). 3D electron microscopy in the physical sciences: The development of Z-contrast and EFTEM tomography. Ultramicroscopy 96, 413-431.

MöBus, G., Doole, R.C. \& InKson, B.J. (2003). Spectroscopic electron tomography. Ultramicroscopy 96, 433-451.

Newbury, D.E. (2005). X-ray spectrometry and spectrum image mapping at output count rates above $100 \mathrm{kHz}$ with a silicon drift detector on a scanning electron microscope. Scanning 27, 227-239.

PANTEL, R. (2011). Coherent bremsstrahlung effect observed during STEM analysis of dopant distribution in silicon devices using large area silicon drift EDX detectors and high brightness electron source. Ultramicroscopy 111, 1607-1618.

ReED, S.J.B. (1975). Electron Microprobe Analysis. Cambridge: Cambridge University Press.

SAghi, Z., Xu, X., Peng, Y., Inkson, B. \& Möbus, G. (2007). Threedimensional chemical analysis of tungsten probes by energy dispersive X-ray nanotomography. Appl Phys Lett 91, 251906.
Schlossmacher, P. (2010). Nanoscale chemical compositional analysis with an innovative S/TEM-EDX system. Microsc Anal 24, S5-S8.

Slater, T.J.A., Camargo, P.H.C., Burke, M.G., Zaluzec, N.J. \& HAIGH, S.J. (2014a). Understanding the limitations of the Super$\mathrm{X}$ energy dispersive $\mathrm{X}$-ray spectrometer as a function of specimen tilt angle for tomographic data acquisition in the S/TEM. J Phys 522, 012025.

Slater, T.J.A., Macedo, A., Schroeder, S.L.M., Burke, M.G., O’Brien, P., CAmargo, P.H.C. \& HaIGH, S.J. (2014b). Correlating catalytic activity of $\mathrm{Ag}-\mathrm{Au}$ nanoparticles with 3D compositional variations. Nano Lett 14(4), 1921-1926.

Tixier, R. \& PHILIBert, J. (1969). Analyse quantitative d'échantillons minces. In Proceedings of the 5th International Congress on X-Ray Optics and Microanalysis, Mollenstedt, G. \& Gaukler, K.H. (Eds.), pp. 180. Berlin: Springer-Verlag.

von Harrach, H.S., Dona, P., Freitag, B., Soltau, H., Niculae, A. \& RoHDE, M. (2010). An integrated multiple silicon drift detector system for transmission electron microscopes. J Phys 241, 012015.

Watanabe, M. (2011). X-ray energy-dispersive spectrometry in scanning transmission electron microscopes. In Scanning Transmission Electron Microscopy: Imaging and Analysis, Pennycook, S.J., Nellist, P.D. \& Peter D. (Eds.), pp. 291-352. New York, NY: Springer Science + Business Media.

Williams, D.B. \& CARTeR, C.B. (2009). Transmission Electron Microscopy: A Textbook for Materials Science, 2nd ed. New York, NY: Springer.

ZALUZEC, N.J. (2014). Analytical formulae for calculation of X-ray detector solid angles in the scanning and scanning/transmission analytical electron microscope. Microsc Microanal 20(1), 1-9. 